\title{
Assessment of the Optic Disc Morphology Using Spectral-Domain Optical Coherence Tomography and Scanning Laser Ophthalmoscopy
}

\author{
Pilar Calvo, ${ }^{1}$ Antonio Ferreras, ${ }^{1,2}$ Beatriz Abadia, ${ }^{1}$ Mirian Ara, ${ }^{1}$ Michele Figus, ${ }^{3}$ \\ Luis E. Pablo, ${ }^{1,2}$ and Paolo Frezzotti ${ }^{4}$ \\ ${ }^{1}$ Ophthalmology Department, Miguel Servet University Hospital, Aragon Health Sciences Institute, \\ Isabel la Catolica 1-3, 50009 Zaragoza, Spain \\ ${ }^{2}$ Department of Surgery, Ginecology and Obstetrics, University of Zaragoza, 50009 Zaragoza, Spain \\ ${ }^{3}$ Department of Neurosciences, University of Pisa, 56126 Pisa, Italy \\ ${ }^{4}$ Department of Ophthalmology, University of Siena, 53100 Siena, Italy
}

Correspondence should be addressed to Antonio Ferreras; aferreras@msn.com

Received 27 February 2014; Accepted 19 June 2014; Published 6 July 2014

Academic Editor: Dan Milea

Copyright (C) 2014 Pilar Calvo et al. This is an open access article distributed under the Creative Commons Attribution License, which permits unrestricted use, distribution, and reproduction in any medium, provided the original work is properly cited.

Objective. To compare the equivalent optic nerve head (OHN) parameters obtained with confocal scanning laser ophthalmoscopy (HRT3) and spectral-domain optical coherence tomography (OCT) in healthy and glaucoma patients. Methods. One hundred and eighty-two consecutive healthy subjects and 156 patients with open-angle glaucoma were divided into 2 groups according to intraocular pressure and visual field outcomes. All participants underwent imaging of the ONH with the HRT3 and the Cirrus OCT. The ONH parameters and the receiver operating characteristic (ROC) curves were compared between both groups. Results. Mean age did not differ between the normal and glaucoma groups (59.55 \pm 9.7 years and $61.05 \pm 9.4$ years, resp.; $P=0.15)$. Rim area, average cup-to-disc $(\mathrm{C} / \mathrm{D})$ ratio, vertical C/D ratio, and cup volume were different between both instruments $(P<0.001)$. All equivalent ONH parameters, except disc area, were different between both groups $(P<0.001)$. The best areas under the ROC curve were observed for vertical C/D ratio ( 0.980 for OCT and 0.942 for HRT3; $P=0.11)$. Sensitivities at $95 \%$ fixed-specificities of OCT parameters were higher than those of HRT3. Conclusions. Equivalent ONH parameters of Cirrus OCT and HRT3 are different and cannot be used interchangeably. ONH parameters measured with OCT yielded a slightly better diagnostic performance.

\section{Introduction}

Identification of damage to the optic nerve head $(\mathrm{ONH})$ is key for glaucoma diagnosis. For more than 10 years, the confocal scanning laser ophthalmoscope Heidelberg Retina Tomograph (HRT, Heidelberg Engineering, Heidelberg, Germany) has been widely used to evaluate the optic disc morphology and monitor changes over time [1-10].

On the other hand, since the introduction of optical coherence tomography (OCT) in 1993 [11, 12], this technique has been rapidly adopted into clinical practice and is now one of the main diagnostic methods in ophthalmology. The OCT is a computer-assisted precision instrument that delineates cross-sectional anatomy of the retina and provides in vivo real time images of different structures of the eye. The performance of OCT has been constantly improved since its introduction, and the latest versions can generate threedimensional images from multiple $A$-scans acquired on the optic disc. The cube of data generated from these $A$-scans enables a far more extensive evaluation of the peripapillary area including retinal nerve fiber layer profiles, en face images (fundus image), and ONH assessment. Diagnostic ability of OCT for glaucoma diagnosis has been reported in the past [13-22]. Nevertheless, few studies have compared the $\mathrm{ONH}$ measurements between these devices [23, 24].

The purpose of this study was to compare the equivalent ONH parameters obtained with Cirrus OCT (Carl Zeiss Meditec, Dublin, CA) and HRT3 in healthy individuals and 
glaucoma patients as well as evaluate their accuracy for glaucoma diagnosis.

\section{Materials and Methods}

The study protocol adhered to the tenets of the Declaration of Helsinki and was approved by the Clinical Research Ethics Committee of Aragón (CEICA).

A total of 350 eyes of 350 subjects were prospectively preenrolled. Normal eyes were consecutively recruited from patients referred for refraction that underwent routine examination without abnormal ocular findings, hospital staff, and relatives of patients in our hospital. The glaucoma group comprised subjects with primary open-angle glaucoma, pseudoexfoliative glaucoma, and pigmentary glaucoma. Patients with glaucoma were recruited consecutively from an ongoing longitudinal follow-up study at the Miguel Servet University Hospital. In 4 cases we could not obtain a reliable standard automated perimetry (SAP) after 3 attempts and in 8 cases the subject did not complete the visits included in the study protocol. These 12 subjects were excluded from further analysis. Finally, 338 eyes of Caucasian origin were included in the statistical analysis. When both eyes fulfilled the inclusion criteria, only one eye per subject was randomly selected.

All of them had to meet the following inclusion criteria: best-corrected visual acuity (BCVA) better than 20/30 (Snellen), refractive error less than 5 spherical diopters and 2 diopters of cylinder, transparent ocular media (nuclear colour/opalescence, cortical or posterior subcapsular lens opacity $<1$ ) according to the Lens Opacities Classification System III system [25], and open-anterior chamber angle. Subjects with previous intraocular surgery, diabetes or other systemic diseases, history of ocular or neurologic disease, or current use of a medication that could affect visual field sensitivity were excluded.

Participants underwent full ophthalmologic examination: clinical history, best-corrected visual acuity, biomicroscopy of anterior segment using a slit lamp, gonioscopy, Goldmann applanation tonometry, central corneal ultrasonic pachymetry (OcuScan RxP; Alcon Laboratories Inc., Irvine, $\mathrm{Ca}$ ), and ophthalmoscopy of the posterior segment.

At least 2 reliable SAPs were performed to minimize the learning effect [26-28]. The visual field was evaluated with a Humphrey Field Analyzer, model 750i (Zeiss Humphrey Systems, Dublin, CA), by using the 24-2 SITA Standard strategy. Near addition was added to the subject's refractive correction. If fixation losses were higher than $20 \%$ or falsepositive or false-negative rates were higher than $15 \%$, the test was repeated. The subjects completed the perimetry measurements prior to any structural test, and each perimetry test was performed at least 3 days apart to avoid a fatigue effect. Abnormal SAP results were defined as typical glaucomatous defects with a pattern standard deviation significantly elevated beyond the $5 \%$ level and/or a Glaucoma Hemifield Test outside normal limits.

The sample was divided into 2 groups according to the intraocular pressure (IOP) and visual field outcome, regardless of optic disc appearance. Glaucomatous eyes had an IOP of greater than $21 \mathrm{mmHg}$ and abnormal SAP results.
Topographic analysis of the optic disc was performed using the HRT3, which provides topographic measurements of the $\mathrm{ONH}$ derived from 16 to 64 optical sections to a depth of $4 \mathrm{~mm}$. The spherical equivalent refractive error of each eye was adjusted in the dioptric ring of the HRT3. Magnification errors were corrected by the software based on keratometric readings. Topographic images were then obtained through dilated pupils (1\% tropicamide eye drops; Alcon Laboratories Inc., Fort Worth, TX) and analysed with the Advanced Glaucoma Analysis 3.1 software. All scans had to have an interscan standard deviation of less than $30 \mu \mathrm{m}$. The margin of the optic disc was manually traced by the same glaucoma specialist, who was masked to the patients' identity and clinical history, defining the inner edge of Elschnig's ring with at least a four-point contour line. All scans had to have an interscan standard deviation of less than $30 \mu \mathrm{m}$. The global stereometric parameters investigated in this study were rim area, disc area, average cup/disc (C/D) ratio, vertical C/D ratio, and cup volume.

The equivalent $\mathrm{ONH}$ parameters were also measured using the Cirrus OCT (Optic Disc Cube $200 \times 200$ scan protocol; software version 6.2) following a standard procedure described elsewhere. Left eye data were converted to a right eye format. All images were acquired with a quality greater than $6 / 10$. The same operator performed all scans with the same device.

All the ophthalmic examinations, perimetry tests, and the topographic analysis of the $\mathrm{ONH}$ were performed within 6 weeks of the subject's date of enrolment into the study.

The statistical analyses were calculated using MedCalc (version 12; MedCalc software, Mariakerke, Belgium) and IBM SPSS statistical software (version 22; IBM Corporation, Somers, NY). All the variables followed a normal distribution as verified with the Kolmogorov-Smirnov test (K-S of 1 sample). Demographics, HRT3, and OCT parameters were compared between both groups with the independent $t$-test.

The receiver operating characteristic (ROC) curves were plotted for the ONH parameters acquired with both devices. The best areas under the ROC curves (AUCs) were also compared (DeLong method) [29].

\section{Results}

Table 1 shows the clinical characteristics of the study sample. Mean age in the normal group (182 eyes) was $59.55 \pm 9.71$ years, while mean age in the glaucoma group (156 eyes) was $61.05 \pm 9.43$ years $(P=0.15)$. There were significant differences $(P<0.001)$ in mean IOP, best-corrected visual acuity, central corneal thickness, mean deviation of SAP, pattern standard deviation of SAP, and the visual field index (VFI) of SAP between both groups.

Disc area measured with Cirrus OCT did not differ between the groups $(P=0.10)$. Nevertheless, HRT3 showed significant differences for disc area between the normal and glaucoma groups $(P<0.001)$. Rim area, average $\mathrm{C} / \mathrm{D}$ ratio, vertical C/D ratio, and cup volume of both Cirrus OCT and HRT3 were different between both groups $(P<0.001)$.

Table 2 shows the equivalent $\mathrm{ONH}$ parameters measured with Cirrus OCT and HRT3 in the normal and glaucoma 
TABLE 1: Clinical characteristics of the sample.

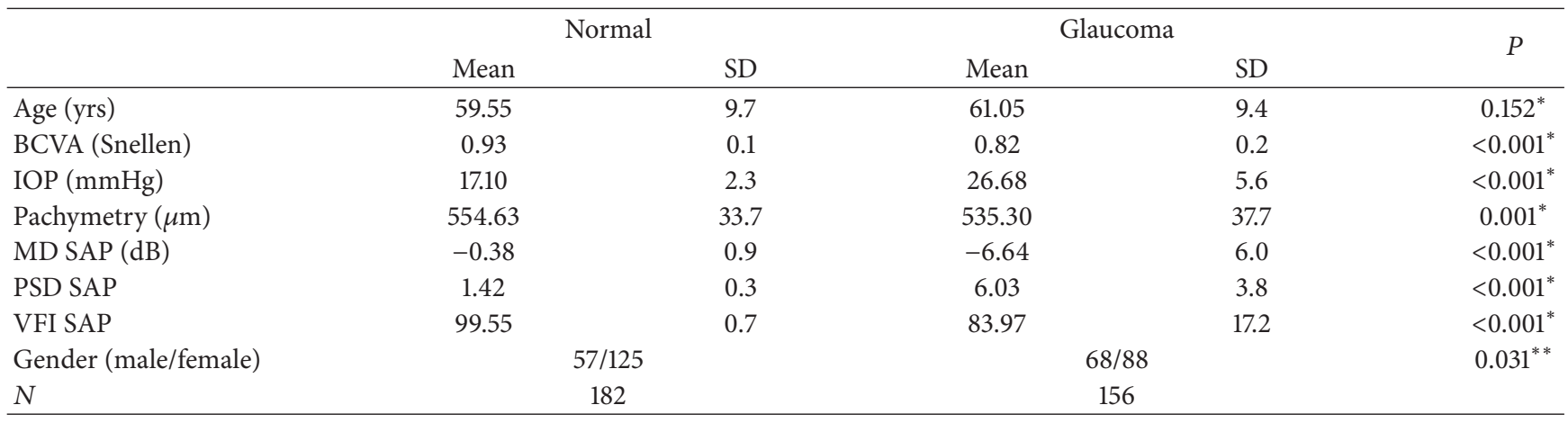

* Student's $t$-test; ${ }^{* *}$ chi-square test; BCVA: best-corrected visual acuity; IOP: intraocular pressure; MD: mean deviation; PSD: pattern standard deviation; SAP: standard automated perimetry; VFI: visual field index; SD: standard deviation; $N$ : number of cases.

TABLE 2: Comparison of the equivalent ONH parameters measured with Cirrus OCT and HRT3 in the normal and glaucoma groups.

\begin{tabular}{lccccccccccc}
\hline & & \multicolumn{4}{c}{ Cirrus OCT } & \multicolumn{4}{c}{ HRT3 } & \multicolumn{2}{c}{$P^{*}$} \\
& & Min & Max & Mean & SD & Min & Max & Mean & SD & 0.32 & 0.87 \\
& & Rim area & 0.87 & 2.33 & 1.49 & 0.29 & 0.86 & 2.59 & 1.50 & 0.32 \\
& Disc area & 1.07 & 2.67 & 1.93 & 0.32 & 0.89 & 3.43 & 1.87 & 0.44 & 0.17 \\
Normal group & Average C/D & 0.06 & 0.77 & 0.42 & 0.17 & 0.00 & 0.58 & 0.19 & 0.13 & $<0.001$ \\
& Vertical C/D & 0.06 & 0.74 & 0.40 & 0.16 & 0.00 & 0.74 & 0.26 & 0.22 & $<0.001$ \\
& Cup volume & 0.00 & 0.78 & 0.10 & 0.13 & 0.00 & 0.48 & 0.07 & 0.09 & 0.050 \\
\hline \multirow{5}{*}{ Glaucoma group } & Rim area & 0.23 & 1.45 & 0.80 & 0.25 & 0.20 & 2.09 & 1.06 & 0.34 & $<0.001$ \\
& Disc area & 1.45 & 2.53 & 2.02 & 0.26 & 1.30 & 3.82 & 2.11 & 0.41 & 0.013 \\
& Average C/D & 0.53 & 0.9 & 0.76 & 0.09 & 0.00 & 0.87 & 0.49 & 0.18 & $<0.001$ \\
& Vertical C/D & 0.54 & 0.89 & 0.74 & 0.09 & 0.00 & 0.95 & 0.66 & 0.21 & $<0.001$ \\
& Cup volume & 0.02 & 0.97 & 0.49 & 0.23 & 0.00 & 1.41 & 0.31 & 0.22 & $<0.001$ \\
\hline
\end{tabular}

* Student's $t$-test.

C/D: cup-to-disc ratio; Min: minimum; Max: maximum; SD: standard deviation.

groups. In the normal group, average and vertical $\mathrm{C} / \mathrm{D}$ ratios were different between Cirrus OCT and HRT3, while in the glaucoma group, all the equivalent $\mathrm{ONH}$ parameters were different $(P<0.001)$.

The largest AUCs obtained with Cirrus OCT (Table 3) were observed for vertical $\mathrm{C} / \mathrm{D}$ ratio $(0.980 ; P<0.001)$ and rim area $(0.966 ; P<0.001)$. All AUCs of Cirrus OCT were above 0.92, except disc area. For HRT3, the largest AUCs were observed for vertical $\mathrm{C} / \mathrm{D}$ ratio $(0.942 ; P<0.001)$ and rim area $(0.905 ; P<0.001)$. The differences of the AUCs between both devices were tested by the DeLong method (Table 4). No differences were found for the best ONH parameters (vertical $\mathrm{C} / \mathrm{D}$ ratio) to discriminate between healthy and glaucoma patients $(P=0.11)$.

Sensitivities of all ONH parameters ranged from $79 \%$ to $98 \%$ at $86 \%$ to $99 \%$ specificities (Table 5 ). The best sensitivityspecificity balance was observed for vertical $\mathrm{C} / \mathrm{D}$ ratio in both devices: $94 \%-93 \%$ for Cirrus OCT and 90\%-94\% for HRT3. The rim area (Cirrus OCT) and vertical $\mathrm{C} / \mathrm{D}$ ratio (HRT3) yielded the highest sensitivities (96.1\%) at 85\% fixedspecificity, whereas the sensitivities were $80.7 \%$ and $91 \%$, respectively, at 95\% fixed-specificity. Overall, sensitivities at 95\% fixed-specificities of the Cirrus OCT parameters were higher than those of HRT3.

\section{Discussion}

This study was aimed at comparing the equivalent $\mathrm{ONH}$ parameters obtained by Cirrus OCT and HRT3 (rim area, disc area, average $\mathrm{C} / \mathrm{D}$ ratio, vertical $\mathrm{C} / \mathrm{D}$ ratio, and cup volume) between healthy and glaucoma patients. We found significant differences for average $\mathrm{C} / \mathrm{D}$ ratio, vertical $\mathrm{C} / \mathrm{D}$ ratio, and cup volume in the normal group and for all equivalent $\mathrm{ONH}$ parameters in the glaucoma group between both instruments.

Few articles have compared the $\mathrm{ONH}$ parameters between Cirrus OCT and HRT3. Sato et al. [23] performed a similar study by comparing $\mathrm{ONH}$ parameters from 96 glaucoma patients and 21 healthy subjects. They also found significant differences $(P<0.001)$ in all the studied parameters, although this analysis was performed on the total sample, not for subgroups.

The software of the HRT3 determines a reference plane parallel to the retina surface. The standard reference plane is located $50 \mu \mathrm{m}$ posterior to the temporal ONH margin. The tissue above the reference plane is considered as "rim" while the structure below is considered as "cup." Although the level of the reference plane should not affect the measurement of the disc area, its position has a direct impact on the 
TABLE 3: Areas under the ROC curve of the optic nerve head parameters to discriminate between healthy and glaucoma patients.

\begin{tabular}{|c|c|c|c|c|c|c|}
\hline & & \multirow{2}{*}{ AUC } & \multirow{2}{*}{$\mathrm{SE}$} & \multirow{2}{*}{$P$} & \multicolumn{2}{|c|}{ CI 95\% } \\
\hline & & & & & Inferior limit & Superior limit \\
\hline \multirow{5}{*}{ Cirrus OCT } & Rim area & 0.966 & 0.02 & $<0.001$ & 0.936 & 0.995 \\
\hline & Disc area & 0.584 & 0.05 & 0.120 & 0.481 & 0.688 \\
\hline & Average C/D & 0.961 & 0.02 & $<0.001$ & 0.932 & 0.991 \\
\hline & Vertical C/D & 0.980 & 0.01 & $<0.001$ & 0.960 & 0.999 \\
\hline & Cup volume & 0.924 & 0.03 & $<0.001$ & 0.873 & 0.975 \\
\hline \multirow{5}{*}{ HRT3 } & Rim area & 0.905 & 0.03 & $<0.001$ & 0.849 & 0.962 \\
\hline & Disc area & 0.642 & 0.05 & 0.009 & 0.544 & 0.740 \\
\hline & Average C/D & 0.887 & 0.03 & $<0.001$ & 0.824 & 0.950 \\
\hline & Vertical C/D & 0.942 & 0.03 & $<0.001$ & 0.888 & 0.995 \\
\hline & Cup volume & 0.902 & 0.03 & $<0.001$ & 0.846 & 0.958 \\
\hline
\end{tabular}

C/D: cup-to-disc ratio; AUC: area under the receiver operating characteristic curve; CI: confidence interval; SD: standard error.

TABLE 4: Comparison of the best areas under the ROC curve of the equivalent optic nerve head parameters between Cirrus OCT and HRT3 (DeLong method).

\begin{tabular}{lcccc}
\hline & & & Cirrus OCT & \\
& & Rim area & Vertical C/D ratio & 0.003 \\
HRT3 & Rim area & 0.015 & 0.114 & 0.318 \\
& Vertical C/D ratio & 0.363 & 0.001 & 0.489 \\
\hline
\end{tabular}

C/D: cup-to-disc.

TABLE 5: Best sensitivity-specificity balance of Cirrus OCT and HRT3 to discriminate between normal and glaucomatous eyes.

\begin{tabular}{|c|c|c|c|c|c|c|c|}
\hline & \multirow{2}{*}{$\begin{array}{c}\text { Optimal } \\
\text { cut-off point }\end{array}$} & \multirow{2}{*}{$\begin{array}{c}\text { Sensitivity } \\
(\%)\end{array}$} & \multirow{2}{*}{$\begin{array}{l}\text { Sensitivity } \\
\text { 95\% CI }\end{array}$} & \multirow{2}{*}{$\begin{array}{c}\text { Specificity } \\
(\%)\end{array}$} & \multirow{2}{*}{$\begin{array}{l}\text { Specificity } \\
95 \% \text { CI }\end{array}$} & \multicolumn{2}{|c|}{ Sensitivity at fixed specificity } \\
\hline & & & & & & Specificity $85 \%$ & Specificity $95 \%$ \\
\hline \multicolumn{8}{|l|}{ Cirrus OCT } \\
\hline Rim area & $\leq 1.2$ & 98.1 & $90.1-100$ & 86.3 & $76.2-96.2$ & 96.1 & 80.7 \\
\hline Average C/D & $>0.67$ & 83.3 & $70.7-92.1$ & 98.9 & $92.6-100$ & 85.2 & 84.6 \\
\hline Vertical C/D & $>0.59$ & 94.2 & $84.6-98.8$ & 93.4 & $84.7-97.7$ & 77.5 & 86.5 \\
\hline Cup volume & $>0.263$ & 87.1 & $75.1-94.6$ & 89.0 & $79.5-95.1$ & 91 & 72.4 \\
\hline \multicolumn{8}{|l|}{ HRT3 } \\
\hline Rim area & $\leq 1.16$ & 79.4 & $66.5-89.4$ & 90.1 & $81.2-96.1$ & 83.3 & 68.5 \\
\hline Average C/D & $>0.39$ & 86.5 & $74.7-94.5$ & 94.5 & $86.6-98.5$ & 90.3 & 80.7 \\
\hline Vertical C/D & $>0.55$ & 90.3 & $79.7-96.9$ & 94.5 & $86.6-98.5$ & 96.1 & 91 \\
\hline Cup volume & $>0.14$ & 83.3 & $70.7-92.1$ & 86.3 & $76.2-93.2$ & 81.4 & 59.6 \\
\hline
\end{tabular}

C/D: cup-to-disc ratio; CI: confidence interval.

calculation of the rim and cup areas. OCT measurement does not depend on reference planes and does not require prior manual outlining of disc boundaries, reducing the dependency on operator skill. Additionally, in Cirrus OCT, the disc edge is determined by the termination of Bruch's membrane; thus, the measurement of the rim and cup areas corresponds with the actual anatomy in the same plane as the optic disc. HRT3 does not take into account the inclination of the optical disc as it acquires the images in horizontal planes, leading to a worse performance in tilted discs compared to OCT.

Other authors have evaluated the differences for $\mathrm{ONH}$ parameters obtained by Stratus OCT and HRT [30-32]. Generally, it is accepted that Stratus OCT tended to measure larger areas of the disc than HRT. In our work we did not observe the same trend: disc area was $1.93 \mathrm{~mm}^{2}$ for Cirrus OCT and $1.87 \mathrm{~mm}^{2}$ for HRT3 $(P=0.17)$ in healthy individuals. In the glaucoma group, disc area was $2.02 \mathrm{~mm}^{2}$ for Cirrus OCT and $2.11 \mathrm{~mm}^{2}$ for $\operatorname{HRT} 3(P=0.01)$. By contrast, in the work of Sato et al. [23] disc areas with Cirrus OCT were smaller than those obtained with the HRT $\left(1.97 \mathrm{~mm}^{2}\right.$ versus $\left.2.27 \mathrm{~mm}^{2}\right)$. They included a small sample in the normal group $(n=21)$ and performed the statistical analysis in the whole sample, including normal and glaucomatous eyes. Larger samples usually lead to more precise estimates.

Moghimi et al. [24] also studied the relationship between the ONH parameters measured with Cirrus OCT and HRT3. 
This work included 13 healthy individuals, 21 glaucoma suspects, and 37 patients with established glaucoma. They reported that HRT3 tended to overestimate disc and rim areas compared to Cirrus OCT.

Regarding the $\mathrm{ONH}$ parameters we found statistical differences for all the ONH parameters, except disc area, between the normal and glaucoma groups for both devices. These findings are consistent with previous studies [33-35]. Mwanza et al. [35] also found differences for all ONH parameters, except for disc area. They included 73 patients with glaucoma ( 31 mild, 14 moderate, and 28 severe glaucomas) and 146 healthy patients. They found AUC values ranging from 0.901 to 0.963 for all parameters, except disc area. They also observed, in agreement with our findings, that vertical $\mathrm{C} / \mathrm{D}$ ratio was one of the best parameters to discriminate between healthy and glaucomatous eyes.

The best HRT3 parameter to differentiate between healthy and glaucoma patients was also vertical C/D ratio (0.942). de León-Ortega et al. [36] found a similar result, although their AUC value was lower (0.861). Obviously, the severity of visual field loss has an important influence on imaging instrument sensitivity [27, 37]. More severe disease is associated with increased sensitivity. In our study, most glaucomatous eyes had mild to moderate visual field defects, according to the Hodapp-Parrish-Anderson score [38].

The quality of the data obtained by the imaging devices is influenced by the media opacity, retinal pigment epithelium status, instrument variability, and positioning and centering of the images. Our sample had transparent ocular media, but this condition is not always possible in clinical practice. Clinicians should take into account that the accuracy of the measurements is related to the quality of the images.

\section{Conclusions}

ONH parameters of Cirrus OCT and HRT3 should not be used interchangeably. Optic disc assessment using Cirrus OCT showed a slightly better diagnostic performance than HRT3 to discriminate between healthy and glaucoma patients.

\section{Conflict of Interests}

The authors declare that they have no conflict of interests regarding the publication of this paper.

\section{Acknowledgment}

This paper is supported in part by the Instituto de Salud Carlos III Grant PI1101239.

\section{References}

[1] G. Wollstein, D. F. Garway-Heath, and R. A. Hitchings, "Identification of early glaucoma cases with the scanning laser ophthalmoscope," Ophthalmology, vol. 105, no. 8, pp. 1557-1563, 1998.

[2] N. V. Swindale, G. Stjepanovic, A. Chin, and F. S. Mikelberg, "Automated analysis of normal and glaucomatous optic nerve head topography images," Investigative Ophthalmology and Visual Science, vol. 41, no. 7, pp. 1730-1742, 2000.

[3] B. A. Ford, P. H. Artes, T. A. McCormick, M. T. Nicolela, R. P. LeBlanc, and B. C. Chauhan, "Comparison of data analysis tools for detection of glaucoma with the Heidelberg retina tomograph," Ophthalmology, vol. 110, no. 6, pp. 1145-1150, 2003.

[4] S. Miglior, M. Guareschi, E. Albe', S. Gomarasca, M. Vavassori, and N. Orzalesi, "Detection of glaucomatous visual field changes using the Moorfields regression analysis of the Heidelberg retina tomograph," American Journal of Ophthalmology, vol. 136, no. 1, pp. 26-33, 2003.

[5] A. Ferreras, A. B. Pajarín, V. Polo, J. M. Larrosa, L. E. Pablo, and F. M. Honrubia, "Diagnostic ability of heidelberg retina tomograph 3 classifications. Glaucoma probability score versus moorfields regression analysis," Ophthalmology, vol. 114, no. 11, pp. 1981-1987, 2007.

[6] A. Ferreras, L. E. Pablo, A. B. Pajarín, J. M. Larrosa, V. Polo, and V. Pueyo, "Diagnostic ability of the Heidelberg retina tomograph 3 for glaucoma," American Journal of Ophthalmology, vol. 145, no. 2, pp. 354-359, 2008.

[7] A. Ferreras, L. E. Pablo, J. M. Larrosa, V. Polo, A. B. Pajarín, and F. M. Honrubia, "Discriminating between normal and glaucoma-damaged eyes with the Heidelberg Retina Tomograph 3," Ophthalmology, vol. 115, no. 5, pp. 775-781, 2008.

[8] B. C. Chauhan, D. M. Hutchison, P. H. Artes et al., "Optic disc progression in glaucoma: comparison of confocal scanning laser tomography to optic disc photographs in a prospective study," Investigative Ophthalmology \& Visual Science, vol. 50, no. 4, pp. 1682-1691, 2009.

[9] L. E. Pablo, A. Ferreras, P. Fogagnolo, M. Figus, and A. B. Pajarin, "Optic nerve head changes in early glaucoma: a comparison between stereophotography and Heidelberg retina tomography," Eye, vol. 24, no. 1, pp. 123-130, 2010.

[10] M. Iester, F. Oddone, M. Prato et al., "Linear discriminant functions to improve the glaucoma probability score analysis to detect glaucomatous optic nerve heads: a multicenter study," Journal of Glaucoma, vol. 22, no. 2, pp. 73-79, 2013.

[11] E. A. Swanson, J. A. Izatt, M. R. Hee et al., "In vivo retinal imaging by optical coherence tomography," Optics Letters, vol. 18, no. 21, pp. 1864-1866, 1993.

[12] A. F. Fercher, C. K. Hitzenberger, W. Drexler, G. Kamp, and H. Sattmann, "In vivo optical coherence tomography," The American Journal of Ophthalmology, vol. 116, no. 1, pp. 113-115, 1993.

[13] L. M. Zangwill, C. Bowd, C. C. Berry et al., "Discriminating between normal and glaucomatous eyes using the Heidelberg Retina Tomograph, GDx Nerve Fiber Analyzer, and Optical Coherence Tomograph," Archives of Ophthalmology, vol. 119, no. 7, pp. 985-993, 2001.

[14] F. A. Medeiros, L. M. Zangwill, C. Bowd, and R. N. Weinreb, "Comparison of the GDx VCC scanning laser polarimeter, HRT II confocal scanning laser ophthalmoscope, and stratus OCT optical coherence tomograph for the detection of glaucoma," Archives of Ophthalmology, vol. 122, no. 6, pp. 827-837, 2004.

[15] K. Nouri-Mahdavi, D. Hoffman, D. P. Tannenbaum, S. K. Law, and J. Caprioli, "Identifying early glaucoma with optical coherence tomography," The American Journal of Ophthalmology, vol. 137, no. 2, pp. 228-235, 2004.

[16] D. L. Budenz, A. Michael, R. T. Chang, J. McSoley, and J. Katz, "Sensitivity and specificity of the Stratus OCT for perimetric glaucoma," Ophthalmology, vol. 112, no. 1, pp. 3-9, 2005. 
[17] R. Sihota, P. Sony, V. Gupta, T. Dada, and R. Singh, "Diagnostic capability of optical coherence tomography in evaluating the degree of glaucomatous retinal nerve fiber damage," Investigative Ophthalmology \& Visual Science, vol. 47, no. 5, pp. 20062010, 2006.

[18] F. Mayoral, V. Polo, A. Ferreras, J. M. Larrosa, V. Pueyo, and F. Honrubia, "Diagnostic ability of stratus optical coherence tomography (OCT) in pre-perimetric glaucoma diagnosis," Archivos de la Sociedad Espanola de Oftalmologia, vol. 81, no. 9, pp. 537-544, 2006.

[19] A. Ferreras, L. E. Pablo, A. B. Pajarín, J. M. Larrosa, V. Polo, and F. M. Honrubia, "Logistic regression analysis for early glaucoma diagnosis using optical coherence tomography," Archives of Ophthalmology, vol. 126, no. 4, pp. 465-470, 2008.

[20] L. E. Pablo, A. Ferreras, A. B. Pajarín, and P. Fogagnolo, "Diagnostic ability of a linear discriminant function for optic nerve head parameters measured with optical coherence tomography for perimetric glaucoma," Eye, vol. 24, no. 6, pp. 1051-1057, 2010.

[21] K. R. Sung, D. Y. Kim, S. B. Park, and M. S. Kook, "Comparison of retinal nerve fiber layer thickness measured by Cirrus HD and Stratus optical coherence tomography," Ophthalmology, vol. 116, no. 7, pp. 1264-1270, 2009.

[22] Y. H. Hwang and Y. Y. Kim, "Glaucoma diagnostic ability of quadrant and clock-hour neuroretinal rim assessment using cirrus HD optical coherence tomography," Investigative Ophthalmology \& Visual Science, vol. 53, no. 4, pp. 2226-2234, 2012.

[23] S. Sato, K. Hirooka, T. Baba, and F. Shiraga, "Comparison of optic nerve head parameters using Heidelberg Retina Tomograph 3 and spectral-domain optical coherence tomography," Clinical \& Experimental Ophthalmology, vol. 40, no. 7, pp. 721726, 2012.

[24] S. Moghimi, H. Hosseini, J. Riddle et al., "Measurement of optic disc size and rim area with spectral-domain OCT and scanning laser ophthalmoscopy," Investigative Ophthalmology and Visual Science, vol. 53, no. 8, pp. 4519-4530, 2012.

[25] L. T. Chylack Jr., J. K. Wolfe, D. M. Singer et al., "The lens opacities classification system III," Archives of Ophthalmology, vol. 111, no. 6, pp. 831-836, 1993.

[26] A. Heijl, A. Lindgren, and G. Lindgren, "Test-retest variability in glaucomatous visual fields," The American Journal of Ophthalmology, vol. 108, no. 2, pp. 130-135, 1989.

[27] M. Gonzalez-Hernandez, L. E. Pablo, K. Armas-Dominguez, R. Rodriguez de La Vega, A. Ferreras, and M. Gonzalez De La Rosa, "Structure-function relationship depends on glaucoma severity," British Journal of Ophthalmology, vol. 93, no. 9, pp. 1195-1199, 2009.

[28] P. Fogagnolo, C. Sangermani, F. Oddone et al., "Long-term perimetric fluctuation in patients with different stages of glaucoma," British Journal of Ophthalmology, vol. 95, no. 2, pp. 189-193, 2011.

[29] E. R. DeLong, D. M. DeLong, and D. L. Clarke-Pearson, "Comparing the areas under two or more correlated receiver operating characteristic curves: a nonparametric approach," Biometrics, vol. 44, no. 3, pp. 837-845, 1988.

[30] J. S. Schuman, G. Wollstein, T. Farra et al., "Comparison of optic nerve head measurements obtained by optical coherence tomography and confocal scanning laser ophthalmoscopy," The American Journal of Ophthalmology, vol. 135, no. 4, pp. 504-512, 2003.

[31] M. E. Iliev, A. Meyenberg, and J. G. Garweg, "Morphometric assessment of normal, suspect and glaucomatous optic discs with Stratus OCT and HRT II," Eye, vol. 20, no. 11, pp. 12881299, 2006.
[32] E. M. Hoffmann, C. Bowd, F. A. Medeiros et al., "Agreement among 3 optical imaging methods for the assessment of optic disc topography," Ophthalmology, vol. 112, no. 12, pp. 2149-2156, 2005.

[33] K. R. Sung, J. H. Na, and Y. Lee, "Glaucoma diagnostic capabilities of optic nerve head parameters as determined by cirrus HD optical coherence tomography," Journal of Glaucoma, vol. 21, no. 7, pp. 498-504, 2012.

[34] J. Mwanza, M. K. Durbin, D. L. Budenz et al., "Glaucoma diagnostic accuracy of ganglion cell-inner plexiform layer thickness: comparison with nerve fiber layer and optic nerve head," Ophthalmology, vol. 119, no. 6, pp. 1151-1158, 2012.

[35] J. Mwanza, J. D. Oakley, D. L. Budenz, and D. R. Anderson, "Ability of cirrus HD-OCT optic nerve head parameters to discriminate normal from glaucomatous eyes," Ophthalmology, vol. 118, no. 2, pp. 241-248, 2011.

[36] J. E. de León-Ortega, L. M. Sakata, B. E. Monheit, G. Mcgwin Jr., S. N. Arthur, and C. A. Girkin, "Comparison of diagnostic accuracy of Heidelberg retina tomograph II and Heidelberg retina tomograph 3 to discriminate glaucomatous and nonglaucomatous eyes," American Journal of Ophthalmology, vol. 144, no. 4, pp. 525-532, 2007.

[37] F. A. Medeiros, L. M. Zangwill, C. Bowd, P. A. Sample, and R. N. Weinreb, "Influence of disease severity and optic disc size on the diagnostic performance of imaging instruments in glaucoma," Investigative Ophthalmology and Visual Science, vol. 47, no. 3, pp. 1008-1015, 2006.

[38] E. Hodapp, R. K. Parrish II, and D. R. Anderson, Clinical Decisions in Glaucoma, Mosby, St. Louis, Mo, USA, 1993. 


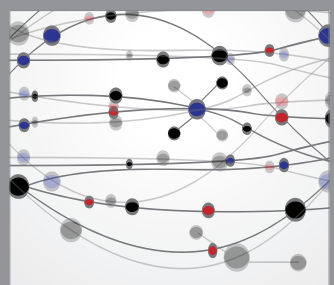

The Scientific World Journal
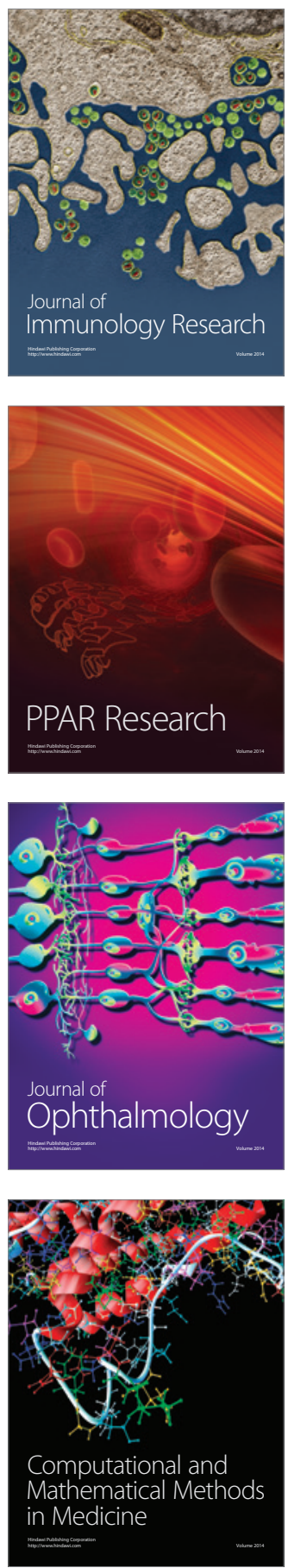

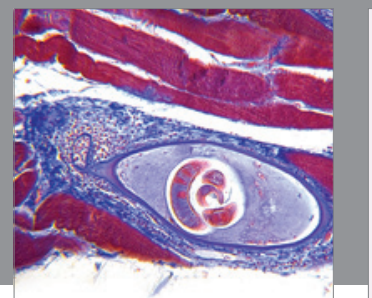

Gastroenterology

Research and Practice
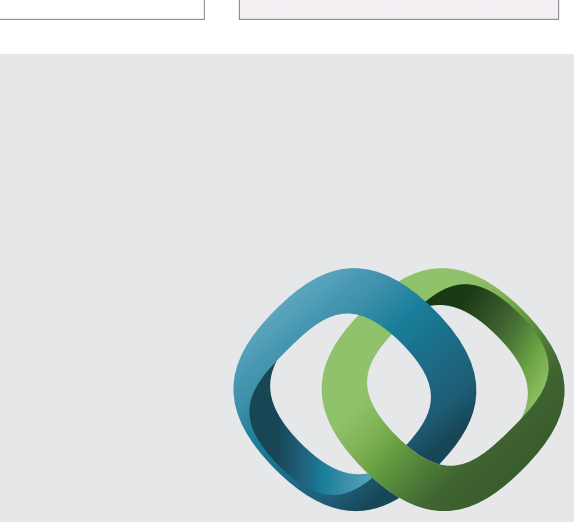

\section{Hindawi}

Submit your manuscripts at

http://www.hindawi.com
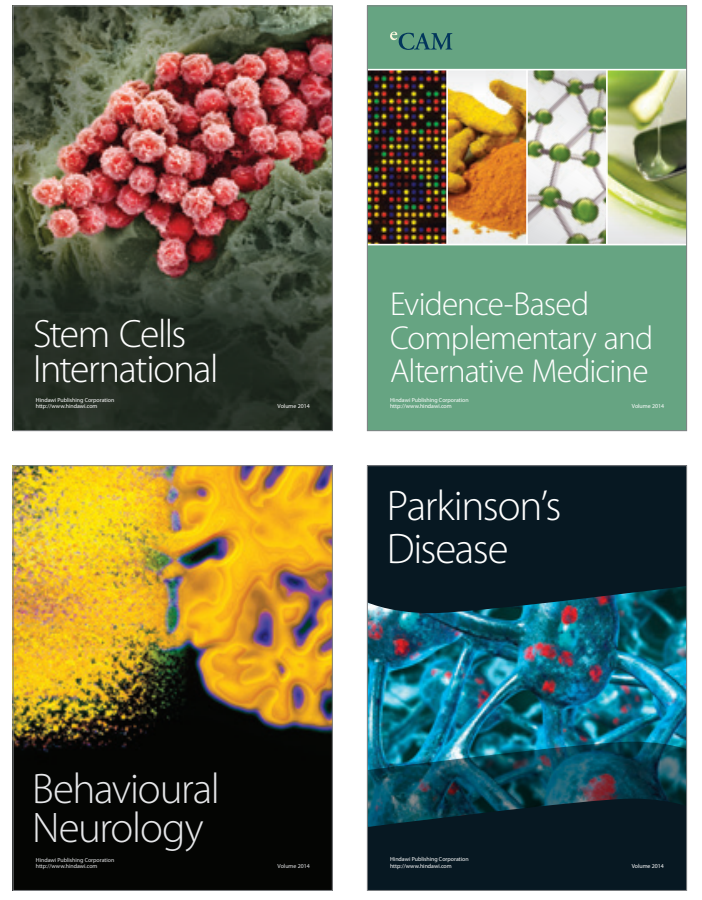
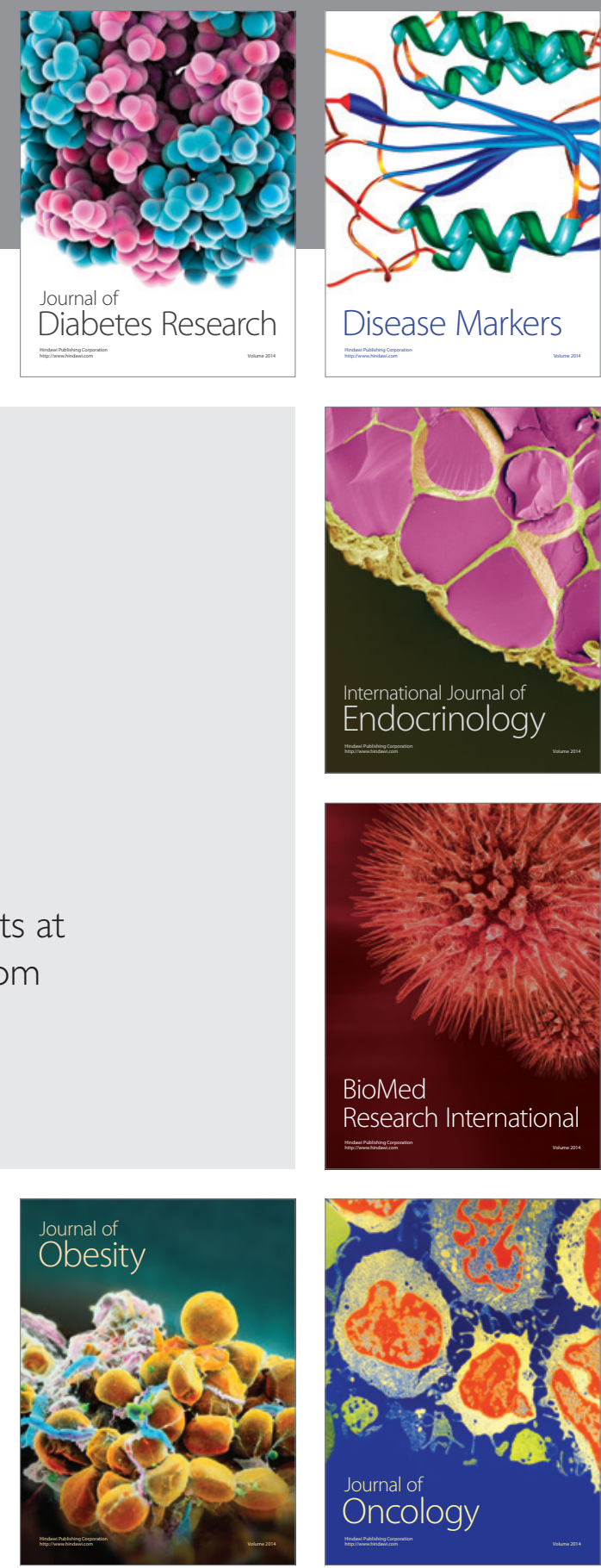

Disease Markers
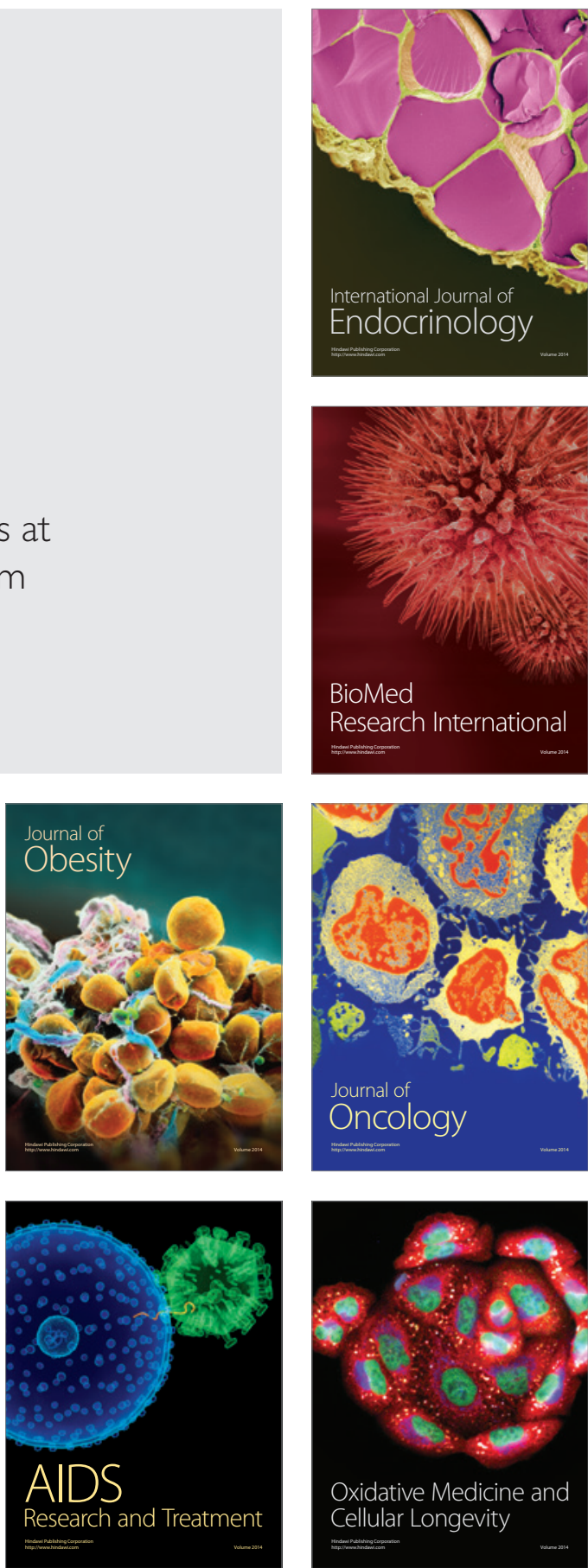